\title{
Optical Design of Enhanced Light Extraction Efficiency in Organic Light Emitting Device with an Optically Controlled Surface Plasmon Coupling
}

\author{
Akiyoshi MIKAMI
}

\author{
Kanazawa Institute of Technology, Department of Electrical Engineering, Faculty of Engineering
}

Received April 26, 2013, Accepted August 22, 2013

This paper is based on the authors' presentation given at the 13th International Symposium on Science and Technology of Lighting (LS-13) held June 24-29, 2012, in Troy, New York, USA.

\begin{abstract}
The light extraction of OLED has been successfully enhanced by the combination of multi-cathode structure and high refractive index intermediate layer, which are introduced to control the surface plasmon coupling in the metal cathode and the distribution of optical energy in OLED, respectively. From the multi scale optical analysis, it was found that an approximately $70 \%$ of electromagnetic energy in the dipole emission was effectively converted to the glass and air modes. The phosphorescent green light emitting device exhibits higher light extraction by a factor of 1.6-1.7.
\end{abstract}

KEYWORDS: organic electroluminescence, light extraction efficiency, surface plasmon, phosphorescence

\section{Introduction}

Organic light-emitting diodes (OLEDs) are now widely recognized as a potential application for the large area flat panel displays and solid state lighting. Internal quantum efficiency of the device has been achieved near $100 \%$ using phosphorescent $(\mathrm{PH})$ materials with proper management of singlet and triplet excitons. However, the external quantum efficiency (EQE) of conventional devices remains $20-30 \%$ because of poor light extraction. One of the reasons is quite a large losses induced by the interaction between the metal cathode and evanescent wave in the near-field of dipole emission ${ }^{122}$. The control of surface plasmon coupling is required in order to use propagation light effectively. In addition, we have already reported that high refractive index substrate make it possible to achieve more than $50 \%$ in out-coupling efficiency by the combination of micro lens array and weak micro-cavity effect ${ }^{3 / 4}$. Although these results are meaningful in an academic sense, high refractive index glass is unsuitable for the mass-production process due to cost and panel size. Another approach will be required for the practical application. In this paper, we demonstrate that the combination of multi-cathode structure and high refractive index interlayer significantly reduces optical losses in waveguide mode and evanescent wave loss, resulting in the increase of air and substrate modes instead. The objective of this work is optical design and analysis of multi-cathode structure for the reduction of surface plasmon loss and for the possibility of enhanced light extraction in OLEDs.

\section{Device structure and preparation}

Figure 1 shows a basic device structure of green $\mathrm{PH}-$ OLED used in this experiment. The device basically consists of an indium-tin-oxide (ITO) bottom electrode, a Poly(3,4-ethylendioxy thiophene)-polystyrenesulfonate (PEDOT : PSS) hole-injection layer, Bis[(1-naphthyl)- $N$ phenyl]benzidine (NPB) hole-transporting layer, a 4,4'$N, N^{\prime}$-dicarbazole-biphenyl (CBP) emissive layer doped with Ir(ppy)3 emitting guest, a 2-(4-Biphenylyl)-5-(4-tertbutylphenyl-1,3,4-oxadiazole) (Bu-PBD) electron transporting layer (ETL) and $\mathrm{LiF} / \mathrm{MgAg}$ cathode including

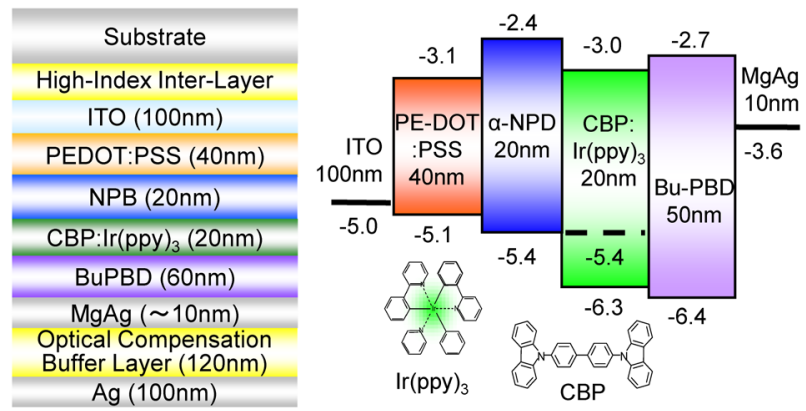

Figure 1 Device structure and potential diagram of phosphorescent OLED.

The device consists of organic layers, a multi-stacked cathode and a high refractive index anode. 
multi-cathode structure behind them. We used titanium-oxide (TiOx) based composite material as a high refractive index medium, which was prepared by simple sol-gel method. Interlayer was formed by spin-coating on the normal refractive index $(n=1.52)$ glass substrate. Multi-cathode structure consists of semitransparent electrode, optically buffer layer (OB-layer) and high reflection mirror layer. The OB-layer was prepared by transparent semi-conducting materials.

\section{Multi scale optical analysis}

Dipole emission from the emitter generally divided into four modes in OLED, namely, external mode, substrate mode, waveguide mode and evanescent mode including direct coupling of near-field with surface plasmon-polariton and lossy surface waves on the metal cathode ${ }^{5}$. If we use an in-plane wave vector, these modes are shown in Figure 2. From the bottom side, the external mode will be treated by classical ray-optics. In the case of substrate mode, we have to take into account a multiple internal reflection in the thick layer. So wave-optics of incoherent light will be suitable for this mode. Waveguide mode should be calculated by electromagnetic optics of coherent light. Surface-Plasmon is directly related to the near-field optics. Wide range of optics is deeply involved with each other in many ways. We have to think about total optical design of the structure and materials for the enhancement of light extraction efficiency. So we used multi scale analysis for the optimization of multi-stacked OLED structure from an optical point of view. We developed an original simulation software named "FROLED" in order to calculate the power spectrum of dipole emission including

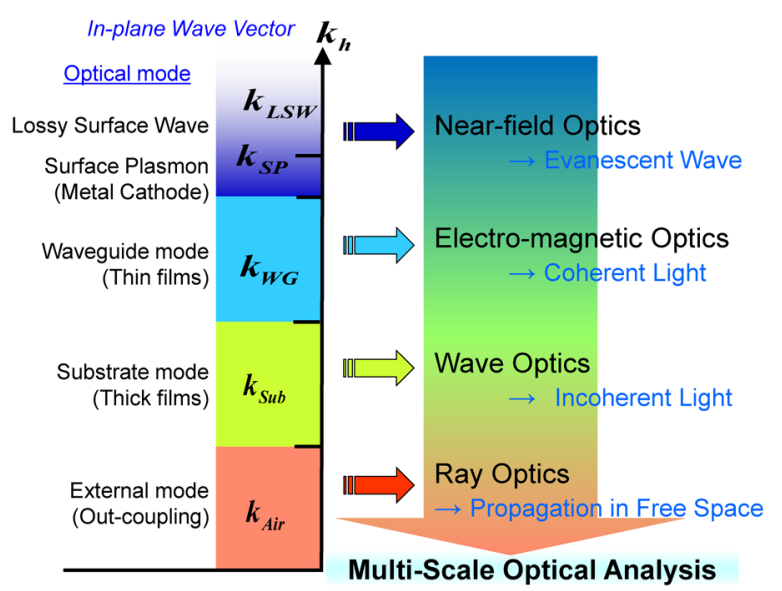

Figure 2 Relationship between various optical modes in OLED and optical theories as a function of in-plane wave-vector.

Since OLED includes wide range of wave-number, a multi scale model is a powerful tool for solving various optical processes produced inside OLED. near-field and far-field optical phenomena in the device. For the enhancement of light extraction, the way of using a substrate propagation mode is much practical to take the light out of the device by conventional micro lens array. However, the sum of external and substrate modes is only less than half (see Figure 5, dashed lines) even if the device is carefully designed because of significant losses induced by waveguide lights and surfaceplasmon coupling. To solve these problems, it will be reasonable to suppress the surface plasmon loss at first, and then to convert an electromagnetic energy in the device from the waveguide mode to the substrate mode, eventually outside the device as an external emission. In this work, three kinds of light extraction techniques were introduced, namely multi-cathode structure on the cathode side, high refractive index intermediate layer on the anode side and micro lens array on the back side of the substrate.

\section{Control of surface plasmon coupling by multi- cathode structure}

Figure 3 shows the percentage of SP-loss in the total electromagnetic energy in OLED as a function of the thicknesses of semitransparent $\mathrm{MgAg}$ cathode and OB-layer. In the thickness range where the cathode layer is as thin as semi-transparency, SP-loss rapidly decreases with the increase of OB-layer. Critical thicknesses of $\mathrm{MgAg}$ layer are about $30 \mathrm{~nm}$. Above those, $\mathrm{MgAg}$ layer has actually high reflection and plasmonic loss mode becomes dominant in spite of the thickness of OB-layer. Figure 4 (a) and (b) are calculated power spectrum in $k$-space for the normal devices with thick $\mathrm{MgAg}$ cathode and a new device with multi-stacked cathode. In the normal device, surface plasmon pole appears at 2.02 of $k$-space in the vertical dipole moment. In contrast, plasmonic mode almost disappears and waveguide TM mode at 1.63 becomes dominant. This means

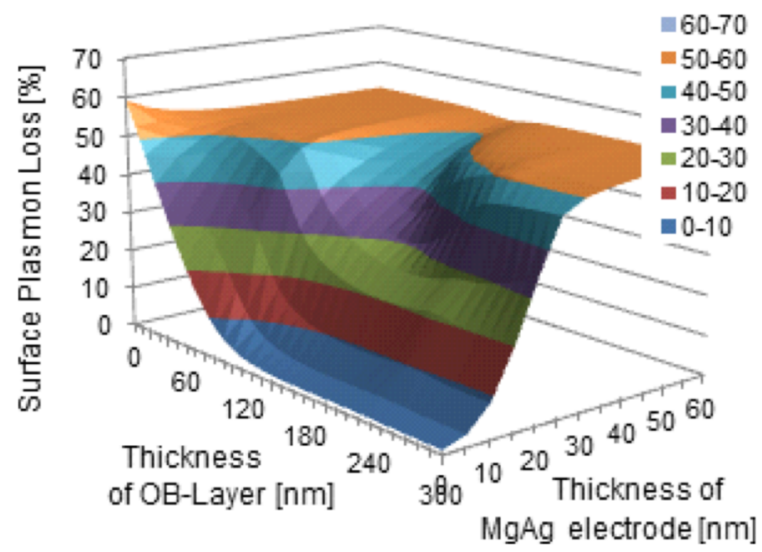

Figure 3 Percentage of surface plasmon loss in total optical energy dissipated in OLED as a function of film thicknesses of MgAg and optical buffer layers. 


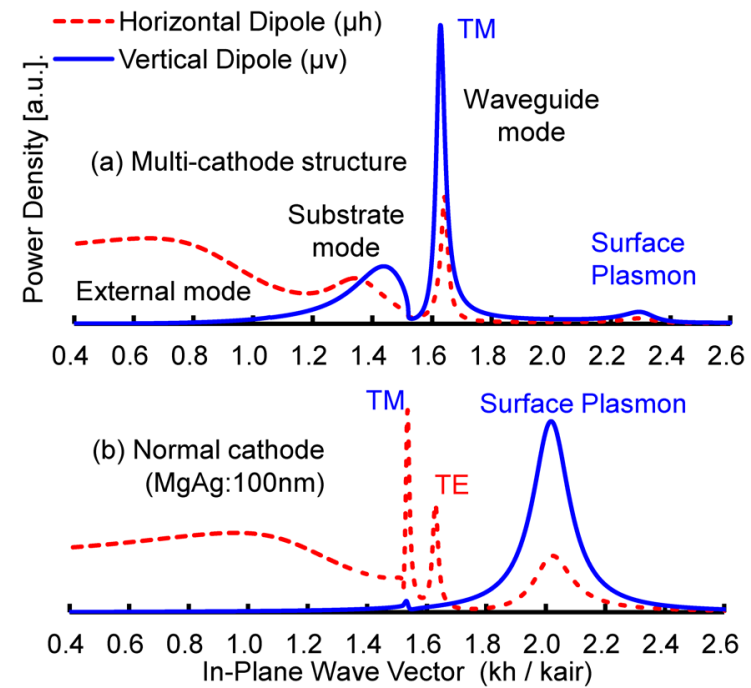

Figure 4 Optical power spectrum of a dipole emission as a function of in-plane wave vector.

(a) Normal device and (b) This work's device with a multi-cathode structure.

that the evanescent wave is successfully converted to the propagation mode by employing the multi-stacked cathode. According to the detail optical analysis, it was found that significant reduction of SP-loss is caused by the interaction between two kinds of SP-coupling on both sides of $\mathrm{MgAg}$ layer, when the film thickness of $\mathrm{MgAg}$ becomes thin so that an evanescent wave of dipole emission reaches the side opposite to $\mathrm{MgAg}$ layer. In that case, the intensity and wave-vector of SP-coupling is supposed to be influenced by optical properties of opposite side of $\mathrm{MgAg}$ cathode. Figure 5 shows the optical energy intensities of multi-modes as a function of the thickness of ETL for two devices with and without multi-stacked cathode structure. The thickness of each layer was fixed to the values shown in Figure 1 except for the ETL. The thicknesses of semitransparent $\mathrm{MgAg}$ and OB-layer are $10 \mathrm{~nm}$ and $140 \mathrm{~nm}$, respectively. The external and substrate modes change periodically with the thickness of ETL. It should be noted that the sum of substrate and external modes takes the first maximum at about $60 \mathrm{~nm}$ in ETL thickness. It is estimated that optical energy intensities were 18\%, 18\%, 10\% and $54 \%$ for external, substrate, waveguide and surface plasmon modes at $60 \mathrm{~nm}$ in the distance between dipole and $\mathrm{MgAg}$ cathode, respectively. Only less than half of total energy is available for out-coupled emission as a propagation light, even if we carefully design the stacked thin-film structure. In the case of new device with multi-stacked cathode, SP-loss is only $12 \%$ and almost all of electromagnetic energy can be converted to propagation waves. Consequently, the intensity of external, substrate and waveguide modes increased to $28 \%, 32 \%$ and $28 \%$, respectively. Especially it should be

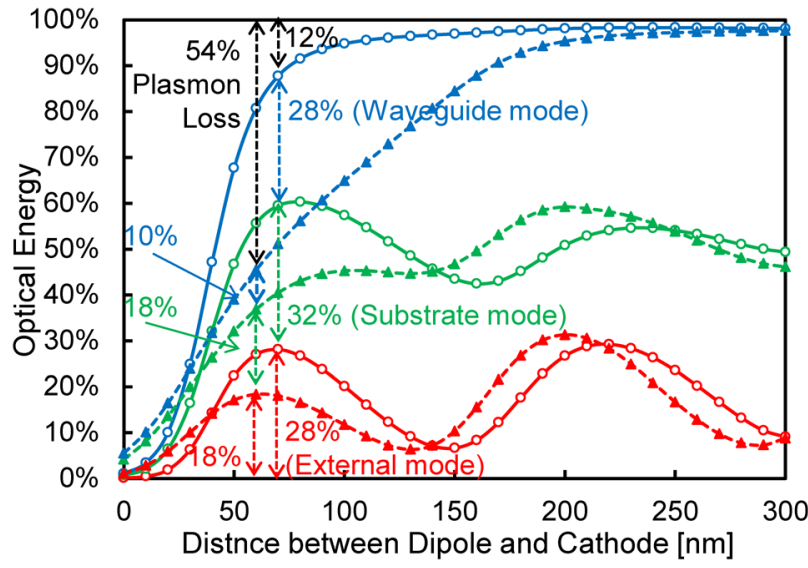

Figure 5 A variation of an electromagnetic energy among external emission, substrate propagation, waveguide mode and surface plasmon loss in OLED as a function of the distance between a dipole and MgAg cathode in the devices with and without a multi-cathode structure.

noted that approximately $90 \%$ of all the electromagnetic energy is converted to the propagation light, and the sum of external and substrate modes reaches to $60 \%$.

\section{Performance of phosphorescent green OLED with high refractive interlayer and micro lens array}

In order to introduce the propagating light in a thin film to a substrate, high index refraction interlayer was introduced between ITO and glass substrate. This layer is a kind of energy converter from the waveguide mode to the substrate mode by means of light scattering effect in high index media. We used titanium oxide (TiOx) based composite material as a high index refraction layer, which was prepared by simple sol-gel method based on the chemical reaction. The oxide film has not only high refractive index but also light scattering effect induced by somewhat porous structure with average pitch size of about $1 \mu \mathrm{m}$ as shown in Figure 6(a). The feature of this layer can be controlled by changing the rotation speed of spin-coating and preparation temperature. The appearance of the film surface is very cloudy, where lights are scattered more strongly.

The use of micro lens array on the backside of the glass substrate is one of the simple and effective techniques for extracting out the substrate mode into air. A configuration of micro lens array is a hexagonal close packed semi-spherical shape as shown in Figure 6(b). The pitch size of the lens array is about $60-80 \mu \mathrm{m}$ in this experiment. Figure 7 shows the comparison of power efficiency between the normal devices and this work's device with multi-cathode structure and high refractive index intermediate layer. The maximum efficiencies of power and EQE are $85 \mathrm{~lm} / \mathrm{W}$ and $23 \%$ in the normal 


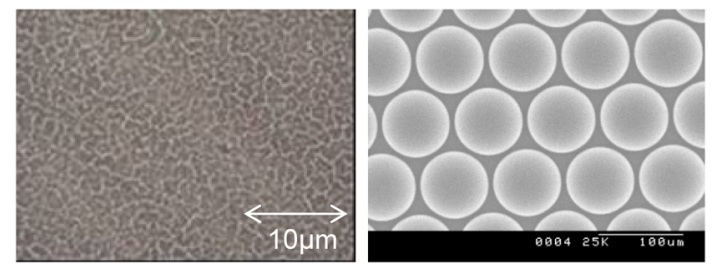

Figure 6 Scanning electron microscope image of surface morphology of high refractive index medium and micro lens array on the glass.

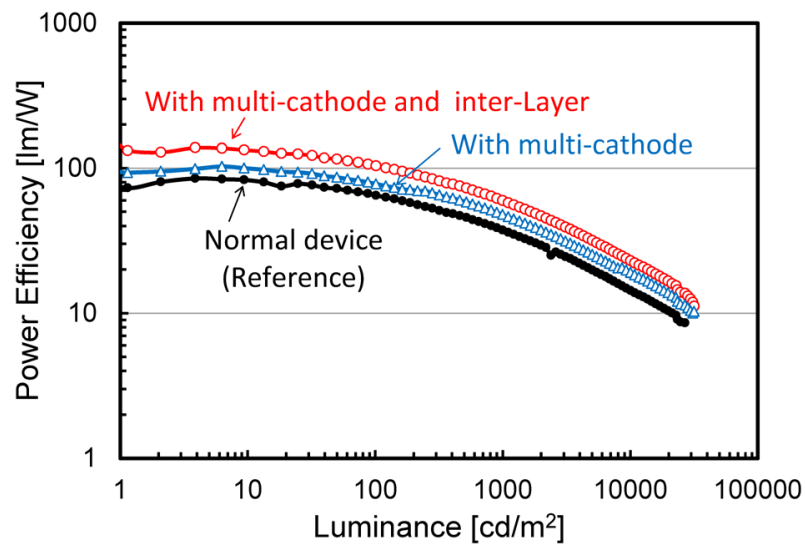

Figure 7 Power efficiency vs. luminance characteristics in three types of OLEDs with and without a multi-cathode structure and a high refractive index interlayer.

green OLED. The power efficiencies of the devices with only multi-cathode and with the both are $102-1 \mathrm{~m} / \mathrm{W}$ and 138-lm/W, respectively. The device showed high power efficiency over $100-\mathrm{lm} / \mathrm{W}$ at $1-100 \mathrm{~cd} / \mathrm{m}^{2}, 50-\mathrm{lm} / \mathrm{W}$ at $100-1,000 \mathrm{~cd} / \mathrm{m}^{2}$ and $25-\mathrm{lm} / \mathrm{W}$ at $10,000 \mathrm{~cd} / \mathrm{m}^{2}$, respectively. EQE of the device was improved to $43 \%$. Device performance will be further improved by optimizing the optical characteristics of multi-cathode structure and high index of refraction interlayer.

\section{Concluding remark}

A multi-cathode structure was introduced to suppress the surface plasmon loss, which increases simultaneously the intensity of an electromagnetic energy in a waveguide mode. As a result, out-coupling efficiency was successfully enhanced by employing the combination with a high-refractive index interlayer and micro lens array. The optically optimized device showed a maximum EQE of $43 \%$ and power efficiencies over $138 \mathrm{~lm} / \mathrm{W}$, which correspond to the improvement by a factor of 1.6-1.7 compared with the reference device. There is a good agreement between EQE and light extraction efficiency from the viewpoint of optical device design. This is resulting from smooth transfer of electromagnetic energy from evanescent wave and waveguide mode to substrate and air modes. Finally we would like to emphasize that a multi scale optical design is effective for the development of enhanced light extraction efficiency in OLED lighting.

\section{References}

(1) Mikami, A.: Digest of Technical Paper, Society for Intern. Display Symposium, San Antonio, pp. 907910 (2009).

(2) Mikami, A.: Phys. Status Solidi C8, No. 9, pp. 28992902 (2011).

(3) Netze, K. A.: J. Opt. Soc. Am., Vol. 15, No. 4, pp. 962-970 (1998).

(4) Chutinan, A., Ishihara, K., Asano, T., Fujita, M. and Noda, S.: Org. Electron., pp. 3-9 (2005).

(5) Bulovic, V., Khalffin, V. B., Gu, G. and Burrowst, P. E.: Phys. Rev. B, Vol. 58, No. 7, pp. 3730-3735 (1998). 